\title{
MEDICIÓN ESTADíSTICA DE LA SATISFACCIÓN DEL CONSUMIDOR EN EL CONSUMO DE CERVEZAS NACIONALES
}

\section{STATISTICAL MEASUREMENT OF THE SATISFACTION OF THE CONSUMER IN THE CONSUMPTION OF NATIONAL BEERS}

Victor Calle Armijos ${ }^{* 1}$, César Freire Quintero ${ }^{2}$, José Luis Chamba Vera ${ }^{3}$, Lisseth Herrera Cedeño ${ }^{3}$, Joselyne Vera Tumbaco ${ }^{3}$

1 Universidad Estatal de Guayaquil, Guayaquil - Ecuador. E-mail: victor.callea@ug.edu.ec

2 Universidad Agraria del Ecuador, Guayaquil - Ecuador. E-mail: cfreire@uagraria.edu.ec

3 Universidad Agraria del Ecuador, Guayaquil - Ecuador. E-mail: joseluistao@hotmail.com, herrera_lisseth@hotmail.com, joselynevera14@hotmail.com

RESUMEN

ABSTRACT

Este estudio busca comparar el grado de satisfacción que produce el consumo de cervezas nacionales de las marcas pilsener, pilsener light y club en los consumidores de la ciudad de Guayaquil, diferenciando la muestra entre hombres y mujeres. Se aplicó el método de encuesta con un alcance de 60 casos. Se recogió la percepción de satisfacción respecto de las tres bebidas antes mencionadas. Se realizó análisis estadístico y se comprobaron los resultados mediante la prueba anova. Entre los resultados se observa que la satisfacción de hombre y mujeres presenta normalidad. En los primeros, y con un grado de significancia de 0.427 se observa que en los hombres no se evidencia una diferencia entre la satisfacción de consumir los tres tipos de cervezas nacionales; mientras que para las mujeres, la prueba arrojó un grado de significancia del 0.045 , demostrando que entre las mujeres sí se evidencia una diferencia entre la satisfacción de consumir los tres tipos de cervezas nacionales.

Palabras Clave: Satisfacción, Cerveza, Guayaquil, Consumidores.
This study seeks to compare the degree of satisfaction produced by the consumption of national beers from the pilsener, pilsener light and club brands in the consumers of the city of guayaquil, differentiating the sample between men and women. The survey method was applied with a scope of 60 cases. The perception of satisfaction regarding the three aforementioned beverages was collected. Statistical analysis was performed and the results were verified by the anova test. Among the results it is observed that the satisfaction of men and women presents normality. In the first, and with a degree of significance of 0.427 , it is observed that in men there is no evidence of a difference between the satisfaction of consuming the three types of national beers; while for women, the test showed a degree of significance of 0.045 , demonstrating that among women there is a difference between the satisfaction of consuming the three types of national beers.

Keywords: Satisfaction, Beer, Guayaquil, Consumers. 


\section{INTRODUCCION}

Conforme en el mundo se destruyen barreras que permiten la igualdad de los géneros, estamos asistiendo a la triste realidad de que el alcoholismo ya ha dejado de ser propio del sexo masculino, sino que también las mujeres lo sufren (Schaffer, 2004). De acuerdo a lo indicado por (Gimenez, 200o), el hígado humano tiene capacidad para eliminar entre 80 y 120 gramos de alcohol al día, en los varones, y unos 50 a 60 en mujeres.

En los países desarrollados, las personas con mejor posición socioeconómica tienden a consumir alcohol de forma regular, pero en cantidades moderadas mientras que los de posición socioeconómica más baja muestran una proporción mayor de abstención (Galán, González, \& Valencia-Martín, 2014). Por otro lado, en países como en Alemania, Holanda, Francia, Suiza, y Austria las mujeres con un elevado nivel educativo consumen más alcohol mientras que los hombres con menor nivel de estudios tienen mayor probabilidad de ser bebedores de riesgo (Bloomfield, Grittner, Kramer, \& Gmel, 2006).

Ensí, elconsumogeneraldealcoholseenlaza con el dominio adquisitivo de la población, ya que del 30\% de la población con el mayor ingreso consume $53 \%$ del alcohol disponible; aunque, el gasto en alcohol es mayor en la población con menos recursos si se considera como proporcional a su ingreso (Medina et al., 2010).

De acuerdo con Carvajal y Zapata (2012) en la decisión de compra de bebidas alcohólicas, las percepciones que cada persona hace del producto, activan la conducta de consumo, independiente de las características físicas o químicas del producto como su sabor o calidad.

Así mismo, en investigaciones realizadas en el país, sobre el comportamiento de los consumidores quiteños al momento de elegir cerveza, se puede resaltar que el principal parámetro para preferir una cerveza va arraigada a su imagen y posicionamiento, en el mercado encontramos cervezas con distintas personalidades y las preferidas son aquellas que captan más la atención de un grupo, ni hombres ni mujeres tienen la capacidad de diferenciar el sabor de una cerveza (Muñoz \& Yépez, 2005).

Es importante destacar que las causas de consumo de sustancias psicoactivas están asociadas a la búsqueda de identidad, de autonomía y de libertad, a nuevas emociones y sensaciones, a mecanismos de escape y a curiosidad (Salcedo, 2011).

De manera específica, lo que más atrae al consumo de alcohol, es la sensación de felicidad (Salcedo, 2011), percepción que ratifica que la cerveza es la bebida más consumida y la motivación psicosocial más frecuente fue la diversión en tiempo libre y los eventos más frecuentados fueron las actividades de grupo y amigos (Campillo \& Vizcay, 2003).

El objetivo de la investigación es Comparar el grado de satisfacción que produce el consumo de cervezas nacionales de las marcas Pilsener, Pilsener Light y Club en los consumidores de la ciudad de Guayaquil, diferenciando la muestra entre hombres y mujeres.

\section{METODOLOGÍA}

El alcance de este estudio es descriptivo, así mismo se considera una lógica deductiva, un enfoque cuantitativo y un diseño de tipo no experimental. Estos criterios de investigación permitieron el desarrollo metodológico de la investigación.

Para este estudio solo se consideraron las marcas ofrecidas por la empresa Cervecería Nacional, considerando a esta la única empresa nacional dedicada a la venta de cervezas nacionales en el país, debido al alto impacto en la economía del Ecuador, que según lo que publicó la revista Líderes "la industria cervecera aporta un $80 \%$ de la recaudación del ICE nacional en bebidas alcohólicas" (Revista Líderes, 2016).

Para la recolección de información respecto a satisfacción de cerveza se usó un cuestionario en la cual se aplicaron escalas de Likert para poder medir percepción referente a ciertos puntos de calidad. Se realizó un muestreo aleatorio en la que se seleccionaron proporcionalmente a hom- 
bres y mujeres consumidores de bebidas alcohólicas. El cuestionario fue sometido a una revisión de expertos para garantizar validez del cuestionario.

Para la confiabilidad del cuestionario se procedió a hacer un muestreo piloto con 30 entrevistados, para lo cual se obtuvo un Coeficiente alfa de Cronbach de 0.822 lo cual recomienda validado el cuestionario a utilizarse, la muestra total fue de 6o individuos proporcionalmente entre hombres y mujeres. No se eliminaron preguntas dado el resultado del coeficiente $\alpha$ de Cronbach, el cual fue suficiente para garantizar la confiabilidad del cuestionario.

Para el análisis de datos se usó la prueba Anova, que es una prueba paramétrica y como tal requiere una serie de supuestos para poder ser aplicada correctamente. La prueba Anova o análisis de la varianza en realidad sirvió para estudiar sus medias y la posibilidad de crear subconjuntos de grupos con medias iguales.

Se desarrolló un análisis estadístico para poder probar las siguientes hipótesis de investigación:

Hipótesis nula: La satisfacción de Pilsener es igual a la satisfacción de Pilsener light y este es igual a la satisfacción de cerveza Club, o sea que los tres tipos de cervezas reflejan el mismo grado satisfac- ción en los consumidores.

Ho: $\mathrm{Sp}=\mathrm{Spl}=\mathrm{Sc}$

Dónde:

La varianza del grado de satisfacción que causa consumir la cerveza Pilsener es igual a la varianza del grado de satisfacción que provoca el consumo de la cerveza Pilsener light y es igual a la varianza del grado de satisfacción que genera el consumo de la cerveza Club.

Hipótesis alternativa: Por lo menos una cerveza tiene una percepción diferente de calidad por parte del consumidor.

$\mathrm{H} 1: \mathrm{Sp} \neq \mathrm{Spl} \neq \mathrm{Sc}$

Dónde:

Al menos la varianza del grado de satisfacción que causa consumir una de los tres tipos de cerveza que se estudia es diferente a la varianza del resto de cervezas.

RESULTADOS

Se procede a realizar las pruebas de normalidad de Kolmogorov-Smirnov y Shapiro-Wilk para los consumidores varones, esto para poder identificar si los valores siguen una distribución normal.

Tabla 1 Prueba de Normalidad

\begin{tabular}{lcccccc}
\hline & \multicolumn{3}{c}{ Kolmogorov-Smirnov } & \multicolumn{3}{c}{ Shapiro-Wilk } \\
\cline { 2 - 7 } & Estadístico & Gl & Sig. & Estadístico & Gl & Sig. \\
\hline Frecuencia Consumo Alcohol & 0,15 & 27 & 0,12 & 0,93 & 27 & 0,07 \\
Frecuencia Baja Graduación & 0,15 & 27 & 0,12 & 0,93 & 27 & 0,07 \\
A Qué Edad Consumes Bebidas & 0,15 & 27 & 0,12 & 0,93 & 27 & 0,07 \\
Alcohólicas & 0,15 & 27 & 0,12 & 0,93 & 27 & 0,07 \\
Satisfacción Cerveza Pilsener & 0,15 & 27 & 0,12 & 0,93 & 27 & 0,07 \\
Satisfacción Cerveza Pilsener Light & 0,15 & 27 & 0,12 & 0,93 & 27 & 0,07 \\
Satisfacción Cerveza club Verde & & & & & &
\end{tabular}

Nota. Sexo = Hombre, Gl: Grados de libertad

La Tabla 1 muestra que los valores de la variable aleatoria dependiente siguen una distribución normal en la población a la que pertenece la muestra, en este caso hombres, ya que tanto el grado de Sig. de Kolmogorov-Smirnov que es o,123 y el de shapiro-wilk que es 0,065, ambos son normales por que superan el o,05.

Una vez que se determinó la normalidad de los valores se procede a realizar el análisis de varianza para el género masculino. 
Tabla 2 Prueba Anova para Grado de Satisfacción

\begin{tabular}{lccccc}
\hline & $\begin{array}{c}\text { Suma de } \\
\text { cuadrados }\end{array}$ & Gl & $\begin{array}{c}\text { Media } \\
\text { cuadrática }\end{array}$ & $\mathrm{F}$ & Sig. \\
\hline Entre grupos & 0,60 & 2 & 0,30 & 0,88 & 0,43 \\
Dentro de grupos & 8,14 & 24 & 0,34 & & \\
Total & 8,74 & 26 & & & \\
\hline
\end{tabular}

Nota. Sexo $=$ Hombre, Gl: Grados de libertad

La hipótesis nula para esta prueba es que la satisfacción de cada una de las cervezas no difiere, responden a una igualdad.

Mientras que la hipótesis alternativa expresa que por lo menos una cerveza tiene una satisfacción distinta a las otras. Con un valor $\mathrm{p}>0,05$ no se rechaza la hipóte- sis nula ya que la satisfacción entre cada una de las cervezas es igual con un nivel de confianza del 95\% (ver Tabla 2).

Esta vez, se procede a realizar las pruebas de normalidad de Kolmogorov-Smirnov y Shapiro-Wilk para las consumidoras mujeres para poder identificar si los valores siguen una distribución normal.

\begin{tabular}{|c|c|c|c|c|c|c|}
\hline & \multicolumn{3}{|c|}{ Kolmogorov-Smirnov } & \multicolumn{3}{|c|}{ Shapiro-Wilk } \\
\hline & Estadístico & $\mathrm{Gl}$ & Sig. & Estadístico & $\mathrm{Gl}$ & Sig. \\
\hline $\begin{array}{l}\text { Frecuencia Consumo } \\
\text { Alcohol }\end{array}$ & 0,15 & 27 & 0,12 & 0,93 & 27 & 0,07 \\
\hline $\begin{array}{l}\text { Frecuencia Baja } \\
\text { Graduación }\end{array}$ & 0,15 & 27 & 0,12 & 0,93 & 27 & 0,07 \\
\hline $\begin{array}{l}\text { A Qué Edad Consumes } \\
\text { Bebidas Alcohólicas }\end{array}$ & 0,15 & 27 & 0,12 & 0,93 & 27 & 0,07 \\
\hline $\begin{array}{l}\text { Satisfacción Cerveza } \\
\text { Pilsener }\end{array}$ & 0,15 & 27 & 0,12 & 0,93 & 27 & 0,07 \\
\hline $\begin{array}{l}\text { Satisfacción Cerveza } \\
\text { Pilsener Light }\end{array}$ & 0,15 & 27 & 0,12 & 0,93 & 27 & 0,07 \\
\hline $\begin{array}{l}\text { Satisfacción Cerveza Club } \\
\text { Verde }\end{array}$ & 0,15 & 27 & 0,12 & 0,93 & 27 & 0,07 \\
\hline
\end{tabular}

Nota. Sexo = Mujer, Gl: Grados de libertad

La Tabla 3 muestra que los valores de la variable aleatoria dependiente siguen una distribución normal en la población a la pertenece la muestra, en este caso las mujeres, ya que tanto el grado de Sig. de Kolmogorov - Smirnov que es o,123 y el de Shapiro-Wilk que es o,o65, superan el o,05.

Una vez que se determinó la normalidad de los valores para el género femenino, se procede a realizar el análisis de varianza.

Tabla 4 Prueba Anova para Grado de Satisfacción

\begin{tabular}{lccccc}
\hline & $\begin{array}{c}\text { Suma de } \\
\text { cuadrados }\end{array}$ & Gl & $\begin{array}{c}\text { Media } \\
\text { cuadrática }\end{array}$ & F & Sig. \\
\hline Entre grupos & 1,49 & 2 & 0,74 & 3,54 & 0,05 \\
Dentro de grupos & 5,03 & 24 & 0,21 & & \\
Total & 6,52 & 26 & & & \\
\hline Nota. Sexo = Mujer, Gl: Grados de libertad & & & &
\end{tabular}

La Tabla 4 evidencia que al 95\% de confianza sí hay una diferenciación, la mujer sí percibe una calidad diferente entre los diferentes tipos de cervezas.

Por ejemplo:
La hipótesis nula es que las mujeres perciben la satisfacción de estos tres tipos de cervezas distintos de la misma manera. Mientras que la hipótesis alternativa es que por lo menos un tipo de cerveza es percibido de manera diferente 
Al 95\% de confianza rechazamos hipótesis nula y se indica que por lo menos una cerveza tiene una percepción diferente.

\section{DISCUSION}

En la investigación del comportamiento del consumidor se resalta la utilización de la metodología mediante el análisis cualitativo y cuantitativo que ayuda a determinar con exactitud el nivel de aceptación de un producto para el consumidor final. El consumidor final es la persona indicada de calificar en forma rápida a las diferentes empresas que ofrecen un bien servicio o un producto es el que exige productos de buena calidad y a precios cómodos (Estupiñan, 2015).

En la mayoría de las sociedades la población adulta tiene un patrón diferenciado de consumo de alcohol según el sexo. Los hombres beben más frecuentemente y en mayores cantidades que las mujeres y como consecuencia tienen más problemas asociados al consumo de alcohol (Anderson \& Baumberg, 2006).

Dos enfoques teóricos se han emplazado para explicar estas diferencias. Uno de ellos enfatiza las diferencias biológicas en la ingesta de alcohol, basándose en el hecho de que las mujeres absorben y metabolizan el alcohol de diferente manera. Principalmente, las mujeres por su menor contenido corporal de agua para un mismo peso, tienen mayores concentraciones de alcohol en sangre después de beber cantidades equivalentes de alcohol, lo que podría influir en beber menos para obtener los mismos efectos (Galán et al., 2014).

Un segundo enfoque destaca las razones culturales y sociales, desde la evidencia de que el consumo de alcohol en las mujeres ha sido sistemáticamente restringido bajo la creencia de que puede afectar adversamente el comportamiento o rol social de la mujer, considerándolo incompatible con las responsabilidades domésticas y signo de fallo del control sobre las relaciones familiares y sociales (Galán et al., 2014).

Los hombres consumen con mayor frecuencia que las mujeres; que la casa es, en segundo lugar, uno de los sitios preferidos para consumir, (lo cual demuestra que el consumo de alcohol está siendo permitido al interior de los hogares); que se consume más cerveza que otros licores quizá por su bajo costo, porque se requiere de mayor cantidad para embriagarse y se tiene menor nivel de censura social sobre el consumo de cerveza que sobre el de aguardiente (Albarracín \& Muñoz, 2008).

Normalmente, cuando se investigan desigualdades en salud, aquellas personas que pertenecen a grupos sociales más desfavorecidos, tienen peor salud y mayor mortalidad que las personas de mayor posición socioeconómica (Collel et al., 2013).

En los países desarrollados, las personas con mejor posición socioeconómica tienden a consumir alcohol de forma regular, pero en cantidades moderadas mientras que los de posición socioeconómica más baja muestran una proporción mayor de abstención, aunque los que beben ingieren mayores cantidades y presentan más problemas relacionados con el consumo. Además, estos patrones varían entre hombres y mujeres. Según un estudio llevado a cabo en 15 países por Bloomfield et al. (2006).

CONCLUSION

En el caso de los hombres, se pudo concluir que la satisfacción que producen los tres tipos de cerveza en ellos es similar.

Para el caso de las mujeres, se pudo concluir que por lo menos uno de los tres tipos de cerveza tiene un nivel de satisfacción diferente en ellas, lo que indica que sí existe una percepción diferente de las mujeres, respecto al grado de satisfacción que producen las cervezas.

\section{REFERENCIAS}

Albarracín, M., \& Muñoz, L. (2008). Factores asociados al consumo de alcohol en estudiantes de los dos primeros años de carrera universitaria. Liberabit, 49-61.

Anderson, P., \& Baumberg, B. (2006). Alcohol in Europe. A. London: Institute 
of Alcohol.

Bloomfield, K., Grittner, U., Kramer, S., \& Gmel, G. (2006). Social inequalities in alcohol consumption and alcohol-related problems in the study countries of the EU concerted action 'Gender, Culture and alcohol problems: a Multi-national Study '. Alcohol, 26-36.

Campillo, R., \& Vizcay, V. (2003). Caracterización del consumo de bebidas alcohólicas en adolescentes de un consultorio médico. Revista Cubana de Medicina General Integral, 45-53 .

Carvajal, J., \& Zapata, E. (2012). Análisis de percepciones de consumidores de bebidas alcohólicas - productos cerveceros. Criterio Libre, 10(17), 107-126.

Colell, E., Sanchez-Niubo, A., \& Domingo-Salvany, A. (2013). Sex differences in the cumulative incidence of substance. Int J Drug Policy.

Estupiñan Medina, J. A. (2o de Noviembre de 2015). Google. Obtenido de Google: http://repositorio.utmachala.edu.ec/ bitstream/4800o/3885/1/ECUACE-2015MKT-CDooo13.pdf

Galán, I., González, M. J. , \& Valencia-Martín, J. (2014). PATRONES DE CONSUMO DE ALCOHOL EN ESPAÑA: UN PAIIS EN TRANSICIÓN. Rev Esp Salud Pública, 529-540.

Gimenez. (2000). Consumo de Alcohol, ALCOHOLISMO. MEDICINA21, 1. Recuperado el 21 de MAYO de 2017, de
https://www.medicina21.com/Articulos-V1143-Consumo_de_alcohol_Alcoholismo.html

Medina, M., García, I., Cortina, D., Orozco, R., Robles, R., Vázquez, L., . . Chisholm, D. (2010). Estudio de costo-efectividad de intervenciones para prevenir el abuso de alcohol en México. Salud Mental 2010;33, Estudio d.

Muñoz, A., \& Yépez, A. (2005). Comportamiento del consumidor quiteño al elegir cervezas : Imagen de marca versus características propias del producto. Quito./

Revista Líderes. (24 de abril de 2016). Las ventas en el sector de la cerveza bajan. Obtenido de http://www.revistalideres.ec: http://www.revistalideres.ec/lideres/produccion-ventas-cerveza-mercado.html.

Salcedo, A. (JUNIO de 2011). Consumo de alcohol en jóvenes universitarios. Redalyc.org, 29(1). Recuperado el 21 de MAYO de 2017, de http://www.redalyc. org/articulo.oa?id=79920065007.

Schaffer, C. (NOVIEMBRE de 2004). ALCOHOLISMO EN LA ADOLESCENCIA. Posgrado de la VIa Cátedra de Medicina, 139. Recuperado el 21 de MAYO de 2017 , de http://med.unne.edu.ar/revista/revista139/alcohol.pdf. 